\title{
Sleep related breathing disorders in adults with Down syndrome
}

\author{
Onofrio Resta', Maria Pia Foschino Barbaro², Tiziana Giliberti', Gennaro \\ Caratozzolo', Maria Grazia Cagnazzo ${ }^{2}$, Franco Scarpelli ${ }^{3}$ and Maria Cristina \\ Nocerino' \\ 'Centre of Sleep Disorders Breathing, Chair of Respiratory Disease, Department of Clinical Methodology and \\ Medical Surgery Technology, University of Bari, Italy \\ ${ }^{2}$ Chair of Respiratory Disease University of Foggia 3 "Casa Divina Provvidenza”, Bisceglie (Bari), Italy
}

\begin{abstract}
Background. While the prevalence of obstructive sleep apnoea syndrome among children with Down syndrome is reported to vary from 30 to $50 \%$, the nocturnal respiratory patterns of adults with Down syndrome is not well known. Objectives. The aim of this study is to evaluate sleep-related breathing disorders in a sample of adults with Down syndrome. Methods. We studied the nocturnal respiratory patterns of 6 adults with Down syndrome, aged 28-53 years. All participants were monitored for 8 hours using a 12 channel polysomnograph. Respiratory events (apnoeic and hypopnoeic) were classified as obstructive or central, in relation to the presence or the absence of paradoxical breathing. Results. All participants had respiratory pauses during sleep. 5 of them had an apnoea/hypopnoea index $>10$, justifying the diagnosis of sleep apnoea syndrome. About $85 \%$ of the respiratory events were apnoeic, the others being hypopnoeic. Among all the respiratory events $89.2 \%$ were obstructive, whereas only $10.8 \%$ were central. The central events were almost always organised in very low and regular sequences and respiration frequently showed a true periodic pattern, consisting of short periods of augmented breath followed by central events causing oxygen desaturation. Conclusions. According to the literature and in conjunction with the current study's results it could be hypothesised that the nocturnal respiratory pattern of adults with Down syndrome depends on several pathogenetic factors such as age, severity of upper airway abnormalities, body mass index (BMI), other pathological conditions and age-related brainstem dysfunction.
\end{abstract}

Keywords: Down syndrome, obstructive sleep apnoea syndrome, central sleep apnoea syndrome, nocturnal respiratory pattern, periodic breathing

\section{Introduction}

The prevalence of obstructive sleep apnoea syndrome among children with Down syndrome, is reported to vary from 30 to $50 \%$ (Stebbens, Denis, Samuels, Croft \& Southall, 1991; Marcus, Keens, Bautista, Von Pechmann \& Ward, 1991; Marcus \& Carol, 1994). This high rate can be explained by the fact that children with Down syndrome often have risk factors for obstructive sleep apnoea syndrome (e.g. midfacial and mandibular hypoplasia, macroglossia and glossoptosis, tonsillar and adenoidal encroachment, obesity, hypothyroidism and generalised hypotonia).

Levanon, Tarasiuk and Tal (1999) found that children with Down syndrome showed sleep abnormalities charac- terized by increased fragmentation, frequent awakenings and arousals and periodic leg movements which were only partially related to obstructive sleep apnoea syndrome. On the other hand, Ferri et al. (1997) recently showed that, among people with Down syndrome without risk factors for obstructive sleep apnoea syndrome, it still remains very frequent. Finally, Telakivi, Partinen, Salmi, Leinonen and Harkonen (1987) found that nocturnal periodic breathing was the main disorder among adults with Down syndrome, and that it was strongly correlated to chronological age. In this paper we studied and evaluated sleep-related breathing disorders in a sample of 6 adults with Down syndrome. 


\section{Method}

\section{Participants}

The participants in the study were 6 adults $(3$ males and 3 females) with Down syndrome (diagnosis confirmed by karyotyping), resident at "Casa della Divina Provvidenza" (a residential facility for people with Down syndrome) in Bisceglie, Bari, Italy.

The demographic characteristics of the participants are shown in Table 1. Only one adult (G.O.) could be considered obese $(\mathrm{BMI}=37.46)$. Neck circumference was measured at the level of the cricothyroid membrane.

None of the participants had any acute diseases (e.g. pulmonary infections, acute bronchitis or acute episodes of respiratory or heart failure). Participants were not treated with sedatives or other drugs.

\section{Measures}

All participants were monitored for 8 hours (22:00-6:00) using a 12 channel polysomnograph (Vitalog HMS 5000, Respironics).

Alpha electroencephalographic activity, electro-oculographic and chin electromyographic recordings were obtained according to standard methods (Rechtscaffen \& Kales, 1968).

Airflow was monitored with thermocouples at the nose and the mouth. Abdominal and ribcage movements were assessed with respiratory inductive plethysmography with recordings of paradoxical breathing.

Night long recordings of oxyhaemoglobin saturation were obtained by finger pulse oximetry. Snoring sounds were recorded with a microphone attached to the neck, and electrocardiograms and sleeping position were also recorded.

To simplify the analysis, sleep was divided into non-rapid eye movement (NREM) and rapid eye movement (REM) sleep; both were expressed as a percentage of total sleep time. Sleep records were scored according to standard criteria (Rechtscaffen \& Kales, 1968).

A respiratory event was defined as a cessation (apnoea) or a reduction (hypopnoea) of $2 / 3$ in airflow or abdominal ribcage movements lasting 10 seconds and associated with a $2-5 \%$ decrease in oxygen saturation. Apnoeic and hypopnoeic events were classified as obstructive in the presence of paradoxical breathing or as central in the absence of paradoxical breathing.

The total number of respiratory events were divided by the total sleep time to obtain the apnoea/hypopnoea index.

The number of events of oxygen desaturation per hour of sleep (oxygen desaturation index) was also calculated.

Baseline arterial oxygen saturation was measured during wakeful periods before sleeping for 5 minutes while the participants were in a supine position.

Periodic breathing was defined as a cyclical fluctuation in breathing with periods of central apnoea/hypopnoea alternating with periods of hyperpnoea in a gradual waxing and waning fashion.

Polysomnography was performed after one night of adaptation in the sleep laboratory.

\section{Results}

Table 2 shows the values measured during polysomnography.

Of the 6 adults with Down syndrome 5 had an apnoea/ hypopnoea index greater than 10 , which can be considered as positive evidence of sleep apnoea syndrome.

About $85 \%$ of the total respiratory events were apnoeic, the others being hypopnoeic. More than one third (36.3\%) of the events occurred during a REM stage, which was only $18.16 \%$ of the total sleep time.

The obstructive sleep apnoeic and obstructive sleep hypopnoeic events were $89.2 \%$ of the total respiratory events, while central sleep apnoeic and central sleep hypopnoeic events formed only $10.8 \%$ and in only one participant (P.A.) were central apnoeic-hypopnoeic events more frequent than obstructive ones.

Central sleep apnoeic and central sleep hypopnoeic events usually occurred in long and regular series, and true periodic respiratory patterns with short periods of hyperventilation alternating with central sleep apnoeic and central sleep hypopnoeic events causing oxygen desaturation were frequent (Figure 1).

Both central sleep apnoeic and obstructive sleep apnoeic events induced significant oxygen desaturation in about $70 \%$ of events.

\begin{tabular}{|l|lllllll|}
\hline Participant & L.M. & D.C. & D.M. & O.A. & P.A. & G.O. & Mean \pm SD \\
\hline Age (years) & 30 & 50 & 53 & 28 & 41 & 30 & $38.66 \pm 10.98$ \\
Sex & F & M & F & F & M & M & \\
Weight (Kg) & 55 & 57 & 50 & 55 & 47 & 90 & $59.00 \pm 15.63$ \\
BMI (Kg/m2) & 24.44 & 24.67 & 27.43 & 22.31 & 20.88 & 37.46 & $26.19 \pm 5.95$ \\
Neck circumference (cm) & 38 & 38.5 & 35 & 35 & 45 & 42 & $38.91 \pm 3.95$ \\
\hline
\end{tabular}

Table I. Demographic characteristics of the participants 


\begin{tabular}{|l|rrrrrrr|}
\hline Participant & L.M. & D.C. & D.M. & O.A. & P.A. & G.O. & Mean \pm SD \\
\hline Total sleep time (min) & 408 & 421 & 503 & 263 & 192 & 419 & $368.66 \pm 115.96$ \\
Rapid Eye Movement \% & 17 & 19 & 9 & 15 & 36 & 13 & $18.16 \pm 9.38$ \\
Apnoea/hypopnoea index & 17 & 36 & 4 & 18 & 24 & 19 & $19.00 \pm 9.20$ \\
Obstructive sleep apnoea events & 83 & 189 & 27 & 62 & 29 & 126 & $86.00 \pm 62.48$ \\
Central sleep apnoea events & 13 & 0 & 0 & 1 & 35 & 0 & $8.16 \pm 14.10$ \\
$\begin{array}{l}\text { Obstructive sleep hypopnoea } \\
\text { events }\end{array}$ & 13 & 40 & 8 & 14 & 0 & 12 & $14.50 \pm 13.50$ \\
Central sleep hypopnoea events & 8 & 0 & 0 & 2 & 14 & 0 & $4.00 \pm 5.79$ \\
Baseline arterial oxygen saturation & 97 & 96 & 97 & 98 & 94 & 89 & $94.66 \pm 3.7$ \\
Oxygen desaturation index & II & II & 5 & 6 & 25 & 25 & $13.83 \pm 8.99$ \\
\hline
\end{tabular}

Table 2. Polysomnographic characteristics of the participants

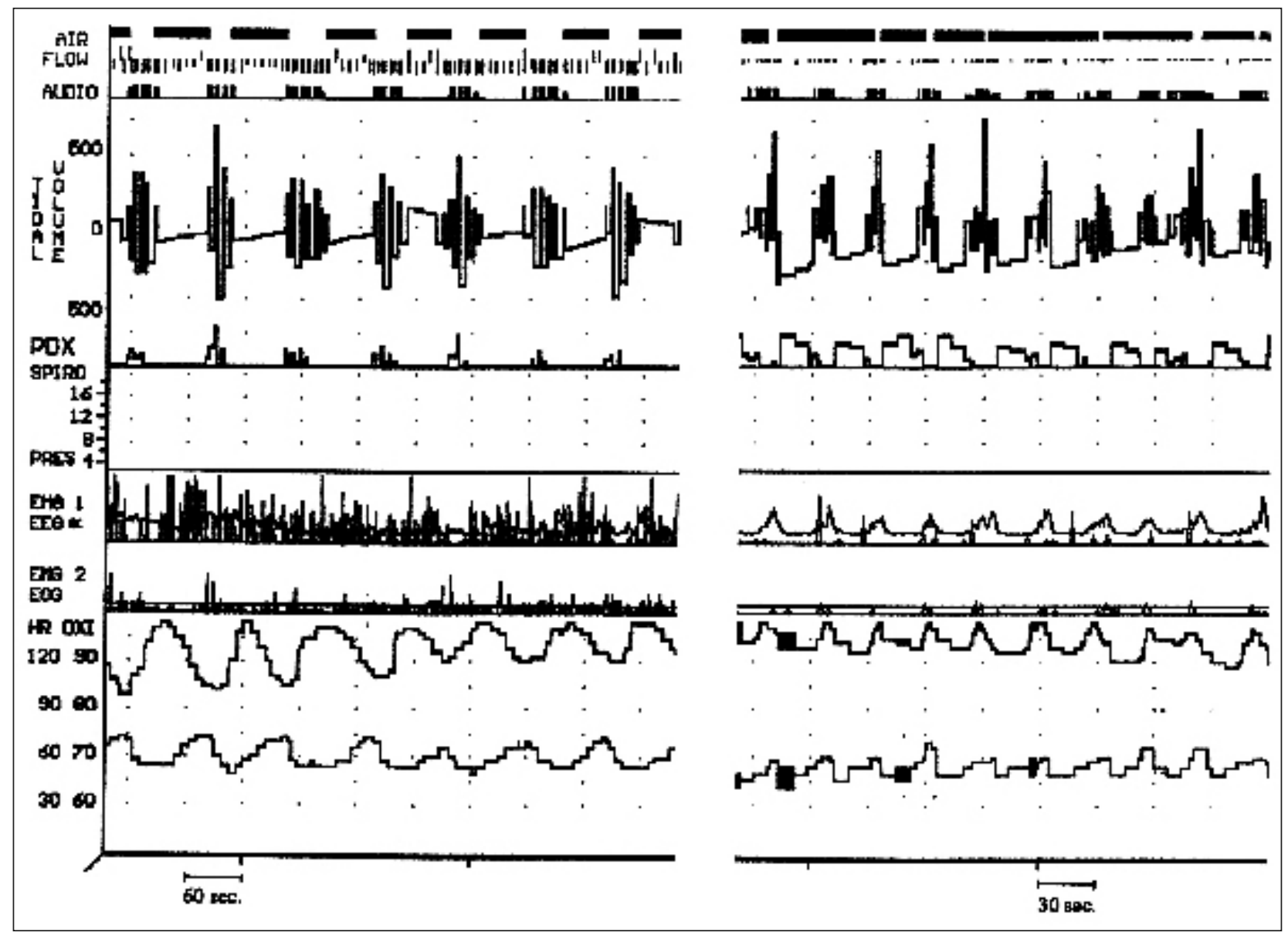

Figure I. Two examples of polysomnographic findings: on the left is representation of a central sleep apnoea during REM sleep stage while that on the right is an obstructive sleep apnoea during non-REM sleep stage. 
Spearman's rank correlation coefficient was calculated to assess the correlation between demographic characteristics, sleep parameters and apnoea/hypopnoea index or oxygen desaturation index.

There was no significant correlation between the apnoea/ hypopnoea index or oxygen desaturation index and age, weight, BMI and neck circumference. As expected, a significant positive correlation was found between baseline arterial oxygen saturation and oxygen desaturation index $($ rho $=.579, \mathrm{p}<.05)$, in spite of the limitations due to the small size of our sample. No significant correlation was found between age and central sleep apnoeic events.

\section{Discussion}

In this paper the nocturnal respiratory patterns of 6 adults with Down syndrome are presented, they were characterised by apnoeic/hypopnoeic events with 5 individuals diagnosed with sleep apnoea syndrome (according to an apnoea/hypopnoea index $>10$ criteria).

Furthermore most respiratory events were obstructive, and in only one participant (P.A.) were they due to central events.

About $30 \%$ of respiratory pauses did not induce a significant oxygen desaturation.

Finally, neither the apnoea/hypopnoea index nor the oxygen desaturation index in these adults were correlated with age or demographic variables.

Many authors (Stebbens et al., 1991; Marcus et al., 1991; Marcus et al., 1994; Southall et al., 1987) have studied nocturnal respiratory patterns in children with Down syndrome. Southall et al. (1987) reported that 6 out of the 12 children with Down syndrome studied presented upper airway obstruction during sleep. Stebbens et al (1991) found, in a sample of 32 children with Down syndrome, a prevalence of obstructive sleep apnoea syndrome of $31 \%$.

Levanon et al. (1999) showed that children with Down syndrome have significant sleep fragmentation, with frequent awakenings and arousals, which are only partially related to obstructive sleep apnoea syndrome.

However, few authors have described the nocturnal respiratory pattern of adults with Down syndrome. Clark, Schmidt and Schuller (1980) observed central sleep apnoeic and obstructive sleep apnoeic events in adults with Down syndrome, finding important upper airway abnormalities in two-thirds of them. Telakivi et al. (1987) found that nocturnal periodic breathing was the most prevalent disorder in a sample of 19 participants with Down syndrome aged 20-65 years, demonstrating that age was the main factor associated with the development of periodic breathing during sleep.

Recently Ferri et al. (1997) described the prevalence of central sleep apnoeic events in a sample of people with Down syndrome (aged 8.6-32.2 years) in the absence of important abnormalities of the upper airways, suggesting that among participants without risk factors for obstruc- tive sleep apnoea syndrome, central sleep apnoeic events frequently occur.

These variable results can be explained by different selection criteria and by the small samples sizes.

In fact, Ferri et al. (1997) studied a selected group without obvious abnormalities of the upper airways, while in the current study the nasopharyngeal status of the individuals was not investigated.

Telakivi et al. (1987) showed that nocturnal periodic breathing was especially present in individuals aged above 40 years (mean age 52.7 years), while the mean age of our participants was 38.6 years. Moreover, Telakivi et al. showed that there was no difference, in terms of severity on the periodical breathing index, between individuals with and without narrowing of the upper airways, and therefore demonstrated that chronological age was the main risk factor for the development of periodical breathing during sleep in people with Down syndrome.

BMI and fat distribution are known risk factors for obstructive sleep apnoea syndrome; in our group the BMI was higher (26.19) than among Ferri's sample (21.6) and Telakivi's subgroup (22.0) and this finding could explain the high prevalence of obstructive events in our sample.

As suggested in the literature, the current study demonstrates that the nocturnal respiratory pattern in adults with Down syndrome is influenced by a number of factors, such as age, upper airway abnormalities, BMI and other additional pathological conditions (e.g. abnormal cardiovascular function).

In the absence of upper airway abnormalities and other pathological conditions and when the BMI is low, age remains the main risk factor for the sleep apnoea syndrome and central sleep apnoeic and central sleep hypopnoeic events become more frequent. Bixler et al. (1985) demonstrated that individuals with Down syndrome, perhaps because of a central cause such as an 'earlier aging process' in their brain, show periodic breathing during sleep at an earlier age than the general population, and this is supported by Telakivi's data. This mechanism could explain the high prevalence of periodic breathing during sleep among individuals with Down syndrome in the absence of risk factors for obstructive sleep apnoea syndrome.

In conclusion, this study shows that among this small study of adults with Down syndrome there is a high prevalence of sleep-related breathing disorders, particularly in terms of obstructive sleep apnoea syndrome. In these individuals the nocturnal respiratory pattern probably depends on several pathogenetic factors such as age, severity of upper airway abnormalities, BMI, other pathological conditions and age-related brainstem dysfunction.

However, the degree of the involvement and the clinical importance of these factors differs and this could explain the different findings in the literature. 


\section{Glossary}

Apnoea - brief interruptions in breathing during sleep.

Body mass index (BMI) $-\mathrm{kg} / \mathrm{m}^{2}$, with an index of 25 or above indicating an individual is overweight, and above 30 indicating obesity.

Central sleep apnoea (CSA) - occurs when the brain fails to send the appropriate signals to the breathing muscles to initiate respirations, and respiratory efforts (e.g. chest movements) stop (cf. obstructive sleep apnoea).

Hyperpnoea - abnormally deep or rapid breathing.

Hypopnoea - a reduction in airflow or abdominal ribcage movements (cf. complete cessation as in apnoea).

Nasopharyngeal - relating to the nose and upper throat.

Obstructive sleep apnoea (OSA) - occurs when airflow is completely or partially, and efforts to breathe continue (cf. central sleep apnoea).

Oxygen saturation - amount of oxygen in blood.

Oxygen desaturation - decreased level of oxygen in blood.

Paradoxical breathing - breathing movements in which the chest wall moves in on inspiration and out on expiration, in reverse of the normal movements.

Periodic breathing - cyclical fluctuation in breathing with periods of central apnoea/hypopnoea, alternating with periods of hyperpnoea.

Polysomnography - recording of a variety of body functions during sleep (such as the electrical activity of the brain, eye movement, chest muscle activity, heart rate, respiratory effort, air flow, and blood gas levels).

\section{Correspondence}

Onofrio Resta • Dipartimento di Metodologia Clinica

e Tecnologie Medico-chirurgiche, Centro Disturbi

Respiratori nel Sonno, Cattedra di Malattie dell'Apparato

Respiratorio, Università di Bari, Piazza G. Cesare, II

- 70124 Bari, Italy • Tel/Fax: +39-080-5566895 • E-mail:

oresta@pneumol.uniba.it

\section{References}

Bixler, E.O., Kales, A., Cadieux, R.J., Vela-Bueno, A., Jacoby, J.A. \& Soldatos, C.R. (1985). Sleep apneic activity in older healthy subjects. Journal of Applied Physiology, 58, 1597-601

Clark, R.W., Schmidt, H.S. \& Schuller, D.E. (1980). Sleep induced ventilatory dysfunction in Down's syndrome. Archives of Internal Medicine, 145, 45-50.

Ferri, R., Curzi-Dascalova, L., Del Gracco, S., Elia, M., Musumeci, S.A. \& Stefanini, M.C. (1997). Respiratory patterns during sleep in Down's syndrome: Importance of central apnoeas. Journal of Sleep Research, 6, 134-41.

Levanon, A., Tarasiuk, A. \& Tal, A. (1999). Sleep characteristics in children with Down syndrome. Journal of Pediatrics, 134, 755-60.
Marcus, C.L., Keens, T.G., Bautista, D.N., Von Pechmann, W.S. \& Ward, S.L. (1991). Obstructive Sleep Apnoea in children with Down's syndrome. Pediatrics, 88, 132-39.

Marcus, C.L. \& Carol, J.L. (1994). Obstructive sleep apnoea syndrome. In G.M. Loughlin \& H. Eighen (Eds.), Respiratory diseases in children: Diagnosis and management (pp. 475-499). Baltimore: Williams and Wilkins.

Rechtschaffen, A. \& Kales, A. (1968). A Manual of Standardized Terminology, Techniques and Scoring System for Sleep Stages of Human Subjects. (NIH publication 204). Washington, DC: US Government Printing Office.

Southall, D.P., Stebbens, V.A., Mirza, R., Lang, M.H., Croft, C.B. \& Shinebourne, E.A. (1987). Upper airway obstruction with hypoxaemia and sleep disruption in Down syndrome. Developmental Medicine and Child Neurology, 29, 734-42.

Stebbens, V.A., Denis, J., Samuels, M.P., Croft, C.B. \& Southall, D.P. (1991). Sleep related upper airway obstruction in a cohort with Down's Syndrome. Archives of Disease in Childhood, 66, 1333-38.

Telakivi, T., Partinen, M., Salmi, T., Leinonen, L. \& Harkonen, T. (1987). Nocturnal periodic breathing in adults with Down's syndrome. Journal of Mental Deficiency Research, 31, 31-9. 